\title{
BMJ Open Insight into the process of postpartum care utilisation and in-home support among vulnerable women in the Netherlands: an in-depth qualitative exploration
}

\author{
Lyzette T Laureij (D) , 'Marije van der Hulst, ${ }^{1}$ Jacqueline Lagendijk (1) , ${ }^{1}$ \\ Jasper V Been, ${ }^{2}$ Hiske E Ernst-Smelt, ${ }^{1}$ Arie Franx, ${ }^{1}$ Marjolein Lugtenberg ${ }^{3}$
}

To cite: Laureij LT, van der Hulst M, Lagendijk J, et al. Insight into the process of postpartum care utilisation and in-home support among vulnerable women in the Netherlands: an in-depth qualitative exploration. BMJ Open 2021;11:e046696. doi:10.1136/ bmjopen-2020-046696

- Prepublication history and additional supplemental material for this paper are available online. To view these files, please visit the journal online (http://dx.doi.org/10.1136/ bmjopen-2020-046696).

Received 10 November 2020 Accepted 25 August 2021

Check for updates

(c) Author(s) (or their employer(s)) 2021. Re-use permitted under CC BY-NC. No commercial re-use. See rights and permissions. Published by BMJ.

${ }^{1}$ Department of Obstetrics and Gynaecology, Erasmus MC, Rotterdam, The Netherlands ${ }^{2}$ Department of Paediatrics, Erasmus MC, Rotterdam, The Netherlands

${ }^{3}$ Department of Public Health, Erasmus MC, Rotterdam, The Netherlands

Correspondence to Dr Marjolein Lugtenberg; m.lugtenberg@erasmusmc.nl

\section{ABSTRACT}

Objective To gain insight into the process of postpartum care utilisation and in-home support among vulnerable women.

Design, method, participants and setting A qualitative interview study was conducted among 23 pregnant and postpartum vulnerable women in the Netherlands, following a grounded theory approach. Women were determined as vulnerable by their healthcare providers. Theoretical sampling of participants was applied and was alternated by data analysis to include information-rich cases until saturation was achieved.

Results A conceptual framework of postpartum care utilisation was generated consisting of three phases: pregnancy, early postpartum period and late postpartum period. Within these phases, information provision, parenting self-efficacy and social network were identified as overarching themes. Perceived inadequate information on content of postpartum care posed a major barrier to forming realistic expectations during pregnancy and hindered its utilisation. Low self-efficacy facilitated postpartum care utilisation. All women experienced increased self-efficacy during and after postpartum care. Support from a social network influenced expectations regarding the added value of postpartum care during pregnancy, and lowered actual utilisation during the postpartum period. The costs of postpartum care and the role of the maternity care assistant acted as general barriers or facilitators influencing the three overarching themes and therefore postpartum care utilisation indirectly.

Conclusions Our findings suggest that postpartum care utilisation among vulnerable women may be improved by considering the particular phase and relevant themes applying to individual women, and adapt care accordingly. We recommend to provide comprehensive, understandable information and to emphasise the gains of postpartum care in improving self-efficacy for vulnerable women. Moreover, involving a woman's social network in postpartum care may add value to this care for this population.
Strengths and limitations of this study

- This study focusses on vulnerable women, a group which is often under-represented in research while having a higher risk of adverse health outcomes.

- We were able to obtain a theoretical sample of vulnerable women needed to conduct a proper qualitative study, resulting in rich and complex data.

- By applying a grounded theory approach, a framework was generated that provides insight into the complex process of postpartum care utilisation.

- Limitations of this study are that views of women who did not use any postpartum care were limited and opinions of certain ethnic minority women may have been missed.

\section{INTRODUCTION}

Women with a low socioeconomic status tend to underuse healthcare services, have an increased risk of adverse health outcomes and are generally less empowered. ${ }^{1-3}$ Where women have multiple medical and nonmedical risk factors for adverse outcomes and a lack of adequate support or coping skills, they are designated as vulnerable. ${ }^{4-6}$ Vulnerability in mothers and their families affects the health and development of their children, which can aggravate inequalities in following generations. ${ }^{7-9}$ Breaking this cycle of inequality by improving healthcare utilisation among vulnerable women might therefore be beneficial for improving health outcomes for next generations.

The early postpartum period is highly suitable for improving health outcomes among mothers and their newborns. ${ }^{10}$ In the Netherlands, postpartum care is provided at home by maternity care assistants (MCAs) during the early postpartum period. MCAs closely monitor the well-being of the mother, the 


\section{Box 1 Postpartum care in the Netherlands}

\section{Provision and content of postpartum care in the Netherlands}

Postpartum care is a unique form of primary care with a focus on prevention of health problems during the postpartum period, provided at home. This care is provided by skilled nurses with a lower secondary education degree, so-called maternity care assistants (MCAs), who are supervised by community midwives.

During pregnancy, every woman may register herself at a chosen maternity care organisation (MCO) in order to receive postpartum care. MCOs are independent enterprises. Their care starts during the third trimester of pregnancy with a home visit by an MCA. This visit is used to explain the content of postpartum care, and to determine the recommended amount of hours of postpartum care during the early postpartum period. This recommendation is based on the Dutch national indication protocol, and is re-evaluated during the first week postpartum by MCAs and midwives. The generally recommended amount of postpartum care is 49 hours (minimal amount is 24 hours, the maximum is 80 hours) spread out over 8-10 consecutive days.

Postpartum care usually starts directly after birth or after discharge from the hospital or birth centre. The provided care from MCAs focusses on information provision, and prevention and identification of healthcare problems, which includes medical check-ups. The information provision is manifold: teaching parents how to take care for their newborn, supporting breast feeding, providing information on what to do when problems occur et cetera. MCAs support parents of newborns with reassurance and positive feedback. During postpartum care, this care is personalised based on the individual needs of the parents. As such, the MCA reduces support as the parents gain confidence in care taking skills. In contract to obstetric care, postpartum care is only partly covered by a woman's mandatory healthcare insurance.

An out-of-pocket payment is required for every hour ( $€ 4.50$ per hour in 2020 ), resulting in an average payment of $€ 220.50$ for the whole period. Some MCOs compensate (a part of) the total out-of-pocket payments for women with a poor financial situation, although there are no protocols for these situations.

newborn and the family, and offer opportunities for prevention of health problems (see box 1). Additionally, MCAs provide women with reliable information about caring for their newborn and provide breastfeeding support. ${ }^{1011}$ Dutch postpartum care is easily accessible and extensive in duration. It is partly covered by obligatory health insurances. ${ }^{12}{ }^{13}$ Whereas approximately $95 \%$ of all postpartum women use at least some amount of postpartum care, utilisation among vulnerable women is lower than among non-vulnerable women. ${ }^{13} 14$ This underuse of postpartum care among vulnerable women is undesirable particularly as these women may benefit most from this preventive and supportive care. ${ }^{15}$

An in-depth understanding of the underlying reasons for the lower utilisation of postpartum care among vulnerable women is currently lacking. By performing a qualitative study, we aimed to gain insight into the process of postpartum care utilisation among vulnerable women, including perceived barriers and facilitators. The results of this study may be used to tailor postpartum care to the needs of vulnerable women, which may subsequently lead to improved utilisation and better health outcomes.

\section{METHODS}

\section{Design}

We used a qualitative design to gain an in-depth understanding of the process of postpartum care utilisation among vulnerable women. A grounded theory approach was deployed, of which the key elements of the alternating process of data collection and analysis, constant comparison and theoretical sampling were applied (see the Selection of participants, Data collection and Data analysis sections for details of this process). This enabled the generation of a conceptual framework grounded in the data. ${ }^{16} 17$

\section{Patient and public involvement statement}

This study aimed to gain insight into the process of postpartum care utilisation among vulnerable women by conducting individual interviews. The study was designed in close collaboration with a council of mothers, healthcare providers and maternity care organisations (MCOs). The study was conducted in collaboration with healthcare providers and MCOs to ensure that outcomes of the study would be relevant for them. All participants were sent their transcript of the interview for member checking. Afterwards, a summary of findings and practical improvements for healthcare providers was communicated in leaflets and symposia.

\section{Research team and reflexivity}

LTL is an experienced qualitative researcher, who previously worked as a physician at an obstetric ward. MvdH is a behavioural scientist with a focus on vulnerable motherchild dyads. ML is an experienced qualitative researcher and psychologist. The analysis of the data was primarily conducted by this multidisciplinary team. In addition, the results of the analysis were discussed with additional members of the research team, being JL (gynaecologist in training), JVB (neonatologist), HEE-S (manager in perinatal care research) and AF (professor in obstetrics and gynaecologist). By involving researchers from different backgrounds, the data were illuminated from different angles.

\section{Selection of participants}

Eligible vulnerable pregnant and postpartum women were approached by different types of healthcare providers during regular care processes: gynaecologists, midwives, MCAs and social workers. Healthcare providers affiliated with the regional consortium 'Pregnancy and Childbirth in the Southwest of the Netherlands' were approached for the inclusion of participants. Women were classified as vulnerable by their healthcare providers based on having a combination of medical and nonmedical risk factors for adverse pregnancy outcomes, and a lack of adequate support or coping skills. ${ }^{4-6}$ Examples 
of medical risk factors are a depression during pregnancy or previous psychiatric diseases. Non-medical risk factors are very broad and may, for example, refer to single motherhood, no fixed abode or financial problems. In addition, women had to be either in their third trimester of pregnancy, to ensure that the home visit of the MCA had taken place, or less than 12 weeks postpartum to prevent recall bias. Women had to be 16 years or older at inclusion, and needed to have a sufficient understanding of the Dutch or English language. Eligible women who were interested in participating, received an informational leaflet including an informed consent form from their healthcare provider.

Theoretical sampling of participants was applied to include information-rich cases until saturation was achieved. In the beginning of the study, all eligible women were invited to participate. Since we interviewed several postpartum women with a Dutch cultural background, we applied deviant case sampling with a shift towards selecting pregnant women. Finally, we specifically selected women based on their lower (intended) use of postpartum care, and women with a non-Dutch cultural background. Single interviews were scheduled with 26 women. Three postpartum women cancelled the interview without giving a reason, and thus seven pregnant and 16 postpartum women were interviewed (table 1). Despite an extensive search, we did not find any women who did not use any postpartum care at all while also meeting our inclusion criteria. After these 23 interviews, 'functional' theoretical saturation was reached (see also the Data analysis) and sampling of interviewees ended.

\section{Data collection}

The interviews were held between January 2018 and November 2018. Interviews were conducted at the participant's home by a trained researcher (LTL) along with a female observer. All interviews started with a short, unrecorded conversation to enable the women to become acquainted with the interviewer and observer. Furthermore, women were reassured that interviews would be handled confidentially. Special consideration was given to simple language use, informal clothing and seating arrangements to encourage women to speak freely and to avoid socially desirable answers. ${ }^{18}$ Women received a 25 euro gift voucher after the interview.

We used an interview guide that was developed based on expert opinions gathered in exploratory meetings with different healthcare providers (ie, MCAs, managers of MCOs and other healthcare providers). Adjustments to the interview guide were made after analysing the first four interviews. The adjusted version focused more on women's experiences regarding postpartum care instead of the providers' opinions (see online supplemental figure S1).

Interviews lasted 27-68 min were audiotaped and transcribed verbatim. All transcripts were checked for accuracy by one of the researchers (LTL or MvdH). The verbatim transcript was sent back to each woman to verify the accuracy and completeness of the text (ie, member check).

\section{Data analysis}

All transcripts were independently analysed by two researchers (LTL and MvdH) using NVivo V.12. The first phase of analysis consisted of open coding of the first twelve transcripts. A preliminary coding scheme was developed and discussed between LTL, MvdH and ML. We selected deviant cases of additional participants based on this coding scheme. Next, axial coding was applied; the data were coded deeper and at a more abstract level and relationships between codes were identified. Codes were grouped together and categories were created. Thereafter, selective coding was applied. Core categories were identified and concepts were created. Overarching themes based on the emergent categories were placed in a conceptual framework grounded in the data and these overarching themes acted as facilitators, barriers or both. In addition, two general facilitators and barriers were identified influencing the three overarching themes and therefore postpartum care utilisation indirectly. The terms facilitators and barriers were applied rather strictly in order to gain insights into the underlying reasons for lower postpartum care utilisation among vulnerable women: further utilisation of postpartum care is stimulated by facilitators independently from the result of this further utilisation, whereas a reason to reduce postpartum care utilisation was defined as a barrier also independently from the result of this reduction of postpartum care utilisation. Constant comparison was applied throughout the whole process of data analysis, by comparing the concepts and emerging framework with new data. 'Functional' theoretical saturation was reached after no new insights for the conceptual framework were identified from the data. ${ }^{19}$

Reporting followed the Standards for Reporting Qualitative Research. ${ }^{20}$

\section{RESULTS}

\section{Conceptual framework}

Women experienced postpartum care as a chronological process started in pregnancy to the postpartum period. This process was divided into three different phases, in which opinions regarding postpartum care were formed and decisions regarding utilisation were made. The first phase was during pregnancy, when women registered at a MCO and the home visit took place. Expectations with respect to the value and decisions regarding utilisation of postpartum care were formed in this phase. The second phase was during the early postpartum period, when women actually experienced postpartum care at home and made decisions about their continued utilisation. The third phase was the late postpartum period. In this phase, reflections on postpartum care were formed regarding the whole postpartum care process. This last 
Table 1 Characteristics of participants

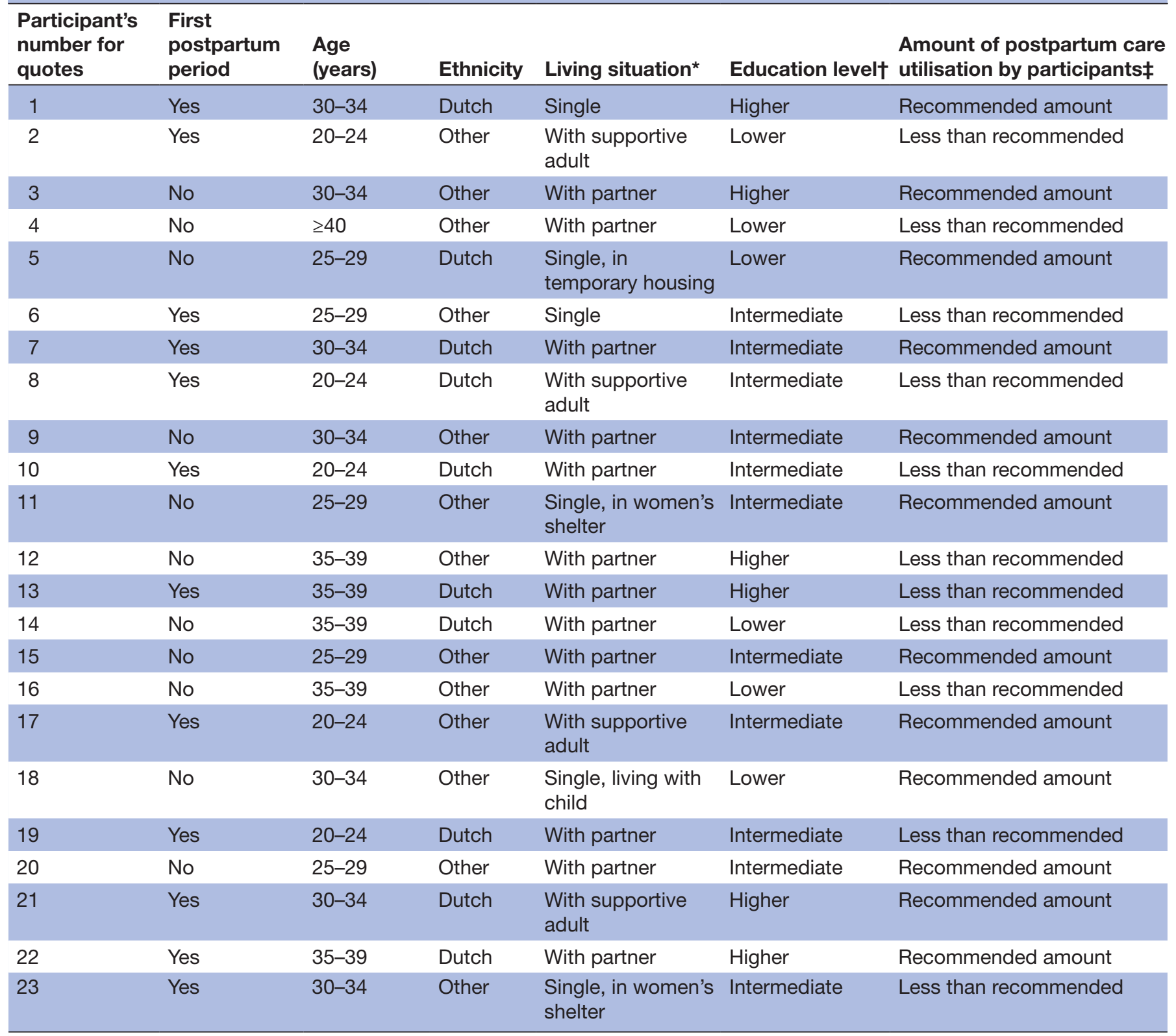

${ }^{*}$ Based on living in a fixed abode, unless indicated otherwise.

†Based on participants' highest successfully completed level of education, classification based on International Standard Classification of Education (ISCED) 2011; lower education corresponds with ISCED levels 0, 1 and 2, intermediate with ISCED levels 3, 4 and 5, and higher with ISCED levels $6,7,8{ }^{42}$

¥For pregnant participants: this represents their intended utilisation of care compared with the recommended amount of hours of postpartum care by their maternity care organisation (MCO). For postpartum participants, this represents their actual use of care compared with the recommended amount of hours postpartum care by their MCO.

phase may ultimately influence decisions about utilisation in a next pregnancy (see figure 1).

We identified three overarching themes influencing the use of postpartum care. These themes are based on what women expressed during the interviews. All themes may act as a facilitator, barrier or both, within each of the three different phases.

1. Information provision: defined as a woman's perception of the provided written and oral information from healthcare providers (including MCAs) regarding the concept and content of postpartum care, and information on newborn caretaking competences provided by MCAs.

2. Parenting self-efficacy: defined as a woman's belief in her own competences to handle difficult situations, such as caring for her newborn. This self-efficacy may differ between the different phases.

3. Social network: defined as a woman's perceived network of persons who may provide practical or emotional support during the postpartum period. This 


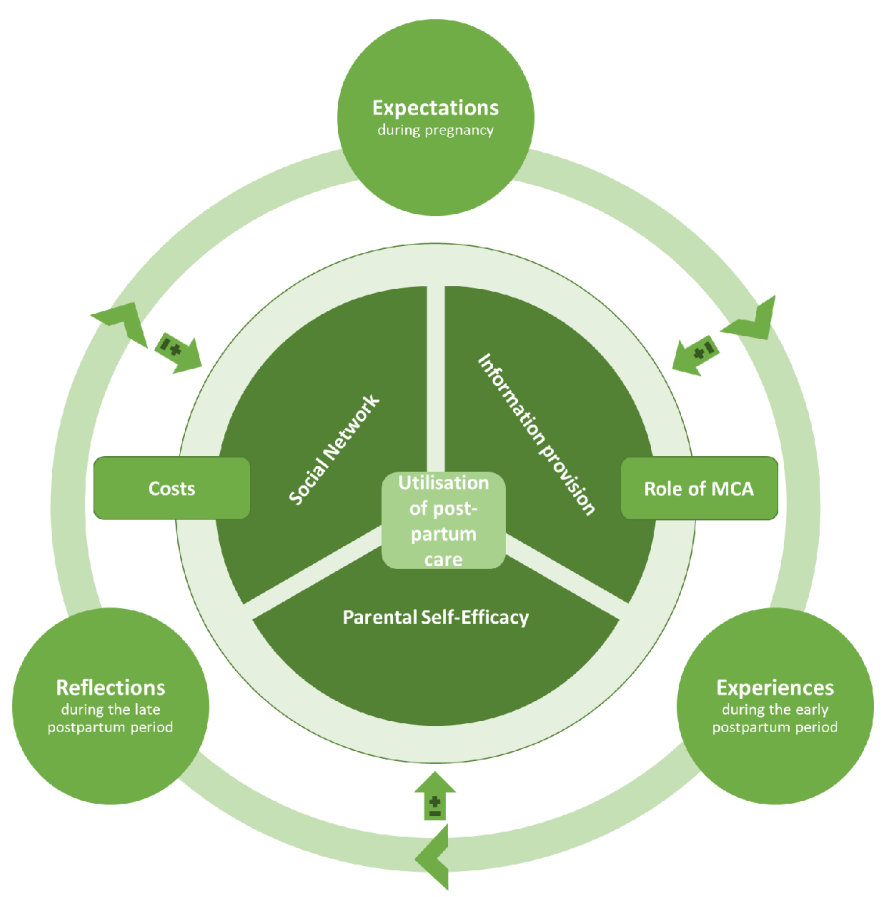

Figure 1 The process of postpartum care utilisation among vulnerable women during pregnancy and the postpartum period. The three phases (outer circle) and three overarching themes (inner circle) influence postpartum care utilisation. Two general facilitators and barriers in the middle circle were also identified to be facilitators or barriers influencing the three overarching themes and therefore postpartum care utilisation indirectly. MCA, maternity care assistants, skilled nurses that provide the postpartum care at home.

network consisted of a combination of their partner (if present), friends and family (eg, mother, mother in law and sister).

Two general facilitators and barriers were identified:

1. Costs of postpartum care: different aspects of costs were identified to be a facilitator or barrier to postpartum care utilisation in the three phases. These different aspects were the concept of the out-of-pocket payments, unawareness of the costs and the total amount of the costs, and the payment of these costs by MCOs in some individual cases.

2. Role of the MCA: different aspects of the role of the MCA acted as a facilitator or barrier to postpartum care utilisation in the three phases. These different aspects were the surveillance, the provision of emotional support, the provision of practical support, the connection with the new parents and the perceived appropriateness of the postpartum care by the new parents.

These general facilitators and barriers were integrated into the description of the different overarching themes per phase.

Below, the framework regarding the use of postpartum care among vulnerable women is described per phase. The different phases, including the perceived barriers and facilitators for the use of postpartum care and the general facilitators and barriers, are set out. Illustrative quotes are provided per theme in the different phases.
Additional supportive quotes can be found in online supplemental table S1.

\section{Expectations of postpartum care during pregnancy}

Women indicated that their expectations regarding the value and utilisation of postpartum care were primarily formed based on information received during pregnancy or during their previous pregnancy and postpartum period (in case of a previous pregnancy). Expectations were furthermore influenced by women's self-efficacy, and their social network.

Information on content of care was provided via leaflets during pregnancy by community midwives or gynaecologists. Although this information provision was perceived as essential for the formation of realistic expectations, information provision via leaflets did not fit women's needs. Additional information was provided during the home visit. Women indicated that they appreciated this visit for getting familiar with their MCO, but did not perceive this as adequate information provision on content of care. Pregnant women stated that lack of adequate information negatively influenced their expectations regarding postpartum care and in some cases resulted in misconceptions; the majority of the women thought that postpartum care was mandatory and some thought that MCAs would place them under surveillance regarding their newborn caretaking competences. Some women also stated that they had no clue as to what MCAs would do all day, and how MCAs would support them:

R: "I just want to, what I need help with, tips so to say, but not 8 hours per day or something... something like that? That is not necessary for me."

I: "And that is because you think that's too much or...?"

R: "Yes, I don't know what they are going to do for eight hours in my house..."

(Respondent \#23, pregnant)

Women with perceived low parenting self-efficacy had high expectations of the benefits of postpartum care during pregnancy, which acted as facilitator for intended utilisation. Especially primiparous women stated that they felt very insecure during pregnancy regarding their skills at newborn care tasks, and therefore expected MCAs to help them with caring for their newborn:

Well, I wanted to have help anyway, and it seemed useful to me as well. I also have to learn a lot about the baby and that [by using postpartum care] is the best way to do it.

(Respondent \#8, postpartum)

Also, the anticipation of not being able to care for their newborn themselves due to complications or fatigue, acted as a facilitator to signing up for postpartum care and forming positive expectations. Women with high levels of parenting self-efficacy generally expected to be able to manage the care for their newborn themselves, 
and wanted MCAs to support them with the household and medical check-ups. Some women with high levels of self-efficacy expected to receive essential and up-to-date information from MCAs, and that this would enhance their self-efficacy even more. Women indicated they wanted to receive all the recommended care if their social network held positive views on it. Some women explained that they were strongly advised by their female friends or relatives to sign up for postpartum care, and followed this advice even though they initially did not want to sign up for postpartum care. Women expected to need less care when they anticipated that their network, including their partner, could help them during the postpartum period:

But that I immediately was like "Expectations?" Don't think about running my household, because that is just my cup of tea and I have my own people for that, so they [MCAs] don't have to come for that.

(Respondent \#14, pregnant)

Women without an extensive social network mostly expected to depend on postpartum care, regardless of having a partner, resulting in a higher intended care utilisation.

\section{Experiences with postpartum care during the early postpartum period}

Experiences with postpartum care were primarily gained during the first week postpartum, and were influenced by the perceived quality of care. In contrast to the expectation of being under surveillance of MCAs, nearly all women felt pampered by their MCAs, and felt positively connected with them. Experiences were again influenced by information provision, self-efficacy and social network which, in turn, acted as barriers or facilitators to care utilisation.

Information was mostly provided orally by MCAs during the early postpartum period. Both primiparous and multiparous women perceived this information as important and felt it was adequately tailored to their individual needs. Hence, adequate information provision acted as a facilitator to continued use of postpartum care. Several women were unaware that out-of-pocket-payments were required for using postpartum care, and decided to reduce the hours of care to what they perceived as necessary, after they gained this information. Others valued postpartum care more than the associated costs and were not deterred by the out-of-pocket-payments into account in their decisions regarding utilisation. A few women considered their own medical status as very complex and felt that MCAs were unable to provide them with the information they needed, and consequently lowered their utilisation. Some women with a non-Dutch cultural background indicated that they followed the advice of their MCA while she was present and that they applied more traditional caretaking techniques with their newborns when the MCA left. This did not affect their care utilisation:
We are used to other things, like uhm, we let the baby sleep in prone position so to say, and yes, here they say like "No, $[\ldots]$ it is not allowed", so uhm, you hear that from other people [...] haha. So yes, I will just, yes I just did that what they told me and then I did what we are used to [after the MCA left].

(Respondent \#11, postpartum)

Experienced low parenting self-efficacy generally positively influenced women's experiences and use of postpartum care. Most women felt insecure during the first days postpartum, and stated they needed all the indicated hours of postpartum care to gain a higher level of selfefficacy. A few women did not like their MCA, but felt too insecure to indicate this to the MCO. Instead of asking for another MCA, they reduced the utilisation of postpartum care. Others stated that they felt secure in caring for their newborn after a few hours of care, and subsequently lowered their postpartum care utilisation because of this increased self-efficacy. All women, including those with high baseline levels of self-efficacy, experienced an increase in self-efficacy due to receiving postpartum care:

And the baby is still small, you can't hold properly, there is something, you miss something. But they also give you the confidence to do that.

\section{(Respondent \#3, postpartum)}

Women who received more support from their social network often asked the MCA to focus solely on the care for the newborn and themselves, while their network supported them in running the household. In some cases this led to a reduction of care utilisation. In contrast, absence of support from a social network facilitated the use of postpartum care, as women felt more dependent on their MCAs:

A sort of, yes, they are your helping hand during such a postpartum period, uhm, but also your support and anchor.

(Respondent \#12, postpartum)

\section{Reflections on postpartum care in the late postpartum period} In this phase, experiences were transformed into reflections regarding postpartum care while looking back on the received care. When reflecting on postpartum care after the early postpartum period, the appraisal of MCAs among women appeared to differ greatly. Whereas most women indicated that they saw MCAs as healthcare providers who provide essential care and support, some felt that MCAs were merely a support in running the household. Similar to the previous two phases, reflections were influenced by women's perception of information provision and their perceived self-efficacy, and social network.

Most women missed information during pregnancy on the fact that MCAs also performed medical check-ups, especially since these medical check-ups were highly appreciated. Women felt that the tailored information 
provided by MCAs postpartum was a huge facilitator for postpartum care utilisation, also for a next postpartum period. Many women did not understand why they needed to pay an out-of-pocket payment for postpartum care at all. However, they generally stressed that they valued the gains of postpartum care over the costs:

R: "Obligatory deductible excess is very common*, you know. That will not change. So yes, if you want the baby to stay healthy and yourself as well, I would definitely recommend it. I don't care about the money, I care more about the uhh..."

I: "The baby? That is important."

R: "The baby."

(Respondent \#16, postpartum)

*There is an obligatory deductible excess for healthcare utilisation in the Netherlands for regular care. Healthcare insurance covers costs above this obligatory deductible excess. However, this does not apply to primary care such as care from a general practitioner, midwife or postpartum care. Obligatory deductible excess is not the same as out-of-pocket payments. Out-of-pocket payments only apply to postpartum care and is independent of the obligatory deductible excess.

Women for whom the out-of-pocket payment was compensated by their MCO stated that this did not affect their postpartum care utilisation.

Most women experienced their increased parenting selfefficacy as one of the most important gains of postpartum care. This increased self-efficacy positively influenced their reflections, and as such affected their intended use of postpartum care following a next postpartum period. Women who already experienced high self-efficacy prior to receiving postpartum care, underlined that they still would use postpartum care in future postpartum periods. They believed this care to be important to monitor the health of their newborn and themselves, and this increased their self-efficacy even more:

... How do I do that? In fact you know how to do it, but you want that extra reassurance that she [maternity care assistant] says like "you are doing great, you just have to do it like this, you can do it.", I just really needed that. And see, she is gone and you need to do it by yourself and you just manage, but I was really insecure like "I can't do it the right way, I might hurt him", or whatever. So she really helped with that.

(Respondent \#9, postpartum)

According to the women, the positive connection that most women experienced with their MCAs contributed to this improved self-efficacy. Postpartum women with a low self-efficacy indicated that they, in hindsight, needed more care than they received but did not dare to express this to their MCA.

Several women specified that they regarded MCAs as essential for providing medical care. They indicated that their social network was not equipped to adequately provide this care. Others indicated that having a sufficient social network during their possible next postpartum period would likely decrease their care utilisation, although they stated that their network could never fully replace MCAs. All women appreciated the provided advice by MCAs regarding caring for their newborns for themselves and their network. Women with a non-Dutch cultural background additionally indicated that MCAs could teach them the newest insights regarding the care for their newborn, as opposed to the traditional way of caring as advised by their mothers and grandmothers:

So I was like... what is really really important to me... uh... in my opinion so to say... in postpartum care is the medical thing for me for example and for the baby as well. Because family is absolutely not capable of doing that [laughs]... you understand?

(Respondent \#4, postpartum)

\section{DISCUSSION}

This qualitative study shows that postpartum care utilisation among vulnerable women is a three-phase process which is mainly influenced by women's perception of the provided information regarding the content of care and actual provided oral information during the provided care, their perceived parenting self-efficacy and perceived support from their social network. During pregnancy, the provided information was perceived as inadequate and posed a major barrier in forming realistic expectations of postpartum care. The information provision during the early postpartum period was found to be essential and this promoted utilisation of postpartum care in a following pregnancy. Low self-efficacy facilitated increased use of postpartum care, which in turn generally improved women's self-efficacy. Women's social network primarily influenced their expectations regarding the added value of postpartum care during pregnancy, and thus influenced actual utilisation during the postpartum period. Furthermore, costs of postpartum care and the role of the MCA indirectly influenced postpartum care utilisation. Overall, vulnerable women recognised the value of postpartum care after experiencing it, and viewed it as essential care for improving their own and their newborns' health.

A strength of this study is that we focused on vulnerable women; a group often under-represented in research. ${ }^{21-23}$ Despite the recruitment challenges, it is important to involve vulnerable populations in research to improve care and outcomes for this group, particularly as they have a higher risk of adverse health outcomes. ${ }^{23}$ Additionally, we showed that it is possible to obtain a theoretical sample of vulnerable women needed to conduct a proper qualitative study. By applying a grounded theory approach, the generation of a conceptual framework grounded in the data was possible. Instead of searching for answers in predefined directions, analyses were largely inductive, allowing meaning to emerge from the data. ${ }^{24}$ 
Theoretical sampling and constant comparisons between the emerging themes and data contributed strongly to the robustness of our results. ${ }^{19}$ Validity of our results was further strengthened by using a multidisciplinary research team with backgrounds in different specialisms.

Despite efforts undertaken, we were unable to include women who did not use any postpartum care (ie, less than $5 \%$ of the total population), limiting our insights into the considerations of this specific subgroup of women. ${ }^{1314} \mathrm{We}$ did interview one woman who gave birth to her second child and did not use postpartum care in the previous postpartum period because she thought it was not useful. Also, we offered interviews in Dutch and English only. We therefore may have missed the opinions of certain ethnic minority women who did not speak these languages.

Our provided conceptual framework regarding postpartum care utilisation is supported by the qualitative systematic review by Walker et $a l^{25}$ They determined necessities for a successful transition to motherhood in the early postpartum period, and identified four themes: connection between women and midwives, identification of women's individual needs, family and cultural influences, and education and support. Our framework builds on this review by focussing on the practical part of the care that is necessary for this transition, and by indicating that this care may be provided by others than midwives. In addition, our theme 'social network' may be an important addition to this review for prevention of serious adverse health outcomes. Cutrona $e t$ al provided an mediational model of postpartum depressions, in which social support positively influences parenting self-efficacy, which has a preventive effect on development of a postpartum depression. ${ }^{26}$ This indicates that healthcare providers, and MCAs in particular, may recognise the role of the social network and parental self-efficacy even more as important themes in postpartum care utilisation.

Using the terms facilitators and barriers regarding utilisation or further utilisation of postpartum care may suggest that utilisation is the ultimate goal. The terms facilitators and barriers were applied rather strictly in our study; when participants reduced their hours of postpartum care, the contributing factor was defined as a barrier, even when it was of a positive nature. For example, women reduced the amount of hours due to increased self-efficacy, strictly this posed a barrier to further utilisation. However, the contributing factor, that is, increased self-efficacy, may be positive. In practice, optimal circumstances should be created to facilitate informed decision making regarding postpartum care utilisation. Reduced or increased number of hours of postpartum care can be desirable, as long as they fit the needs and requirements of the individual woman and her family.

Women perceived the provided information on content of care during pregnancy as inadequate, even after the home visit. This posed an unexpected major barrier in forming realistic expectations regarding postpartum care and affected (intended) utilisation. Especially the supportive nature of postpartum care was unclear from the information provided. The feeling of surveillance instead of support was found to be a barrier for care utilisation among vulnerable women. ${ }^{27}$ Regardless of their social background, women may find it hard to navigate the overwhelming amount of information available. ${ }^{128-30}$ Health illiteracy may aggravate this experienced lack of adequate information received during pregnancy, and may negatively affect utilisation of postpartum care among vulnerable women. ${ }^{25} 3132$ The highly appreciated, tailored oral information provided by MCAs during the early postpartum period indeed signals the possible influence of health illiteracy. ${ }^{334}$ Also, the requirement for outof-pocket payments made some women decide to reduce postpartum care utilisation. This indicates a substantial need for MCOs and healthcare providers to properly inform this vulnerable population about the value of postpartum care already during pregnancy.

The experienced support of MCAs focussing on newborn care competences increased all women's selfefficacy, and as such improved further utilisation of care. In accordance with previous research, ${ }^{35}$ we found that multiparous women and women who already experienced high levels of self-efficacy still said to have benefitted from the confirmation and support given by MCAs. Previous studies showed that connections with healthcare providers were often problematic among vulnerable populations. ${ }^{27} 3637$ However, we found that most of our participants had a good connection with their MCAs, possibly contributing to the experienced increased selfefficacy. ${ }^{38}{ }^{39}$ This good connection may be grounded in the nature of this care: easily accessible care at home, leading to continuity of care in a turbulent phase. ${ }^{29}$ Nevertheless, some women did not dare to stand up for themselves when postpartum care did not match their needs. This posed an important barrier to utilisation, as these women rather abstained themselves from care than to ask for changes. Emphasising the potential role of postpartum care in increasing women's self-efficacy, and tailored care based on a woman's self-efficacy may be used to improve care utilisation.

Finally, we found that women in absence of a supportive network perceived MCAs as their anchor during the early postpartum period. This may be related to the finding that women in general need companionship, and continuity of care in this period. ${ }^{29}$ Also, women with a supportive network highly appreciated the expertise of the MCAs. Providing women and their social network with up-to-date professional knowledge by MCAs might be beneficial for optimising support for women, particularly in the late postpartum period. ${ }^{3435}$ The assessment of a woman's social network and collaboration with this network by MCAs may lead to improved women-centred care and better care utilisation. ${ }^{11} 4041$

The results of our study indicate that information provision during pregnancy must be improved and tailored to vulnerable populations. This information should highlight the supportive and medical tasks of MCAs, outline the need for out-of-pocket payments and emphasise the potential 
gains such as improved self-efficacy. For example, this could be done by sending a video to women during pregnancy with an explanation of postpartum care and the gains. Addressing these aspects during pregnancy may lead to increased utilisation of postpartum care, which impacts subsequent healthcare expenditure. ${ }^{14}$ Moreover, the provided care should be tailored to a woman's individual needs, with extra attention for women with low self-efficacy. Finally, a woman's social network could be involved in the care for the newborn to maximise support and continuity in support in the late postpartum period. This could be initiated by involving the partner or another relative (eg, mother or aunt) from the home visit onwards and by emphasising his or her importance in the early postpartum period. In the absence of a woman's social network, it is important for MCAs to realise they play an important role in providing support. In countries where there is no extensive postpartum care, this support can also be provided by community midwives or general practitioners. They may use videos with information provision on how to take care of a newborn, and try to involve persons in the mother's network who can support the mother. Future research among vulnerable women should focus on the role of postpartum care in improving self-efficacy, and on the effects of eliminating out-of-pocket payments on the utilisation of this care. ${ }^{14}$ Additionally, this study was performed in a developed country. The issue of underutilisation of postpartum care services in low and middle income countries deserves further exploration in these countries, since women interviewed in this study may have a better support network to overcome problems than others who live in less favoured countries.

\section{CONCLUSION}

Our study shows that postpartum care utilisation among vulnerable women is a three-phase process in which information provision, perceived self-efficacy and social network act as either barriers or facilitators to postpartum care utilisation. Individual assessment of these themes and their influence during pregnancy and the whole postpartum period is therefore essential in order to tailor care to vulnerable women's needs and improve utilisation. In general, providing understandable information highlighting the supportive and medical tasks of MCAs, possible gains in improving self-efficacy and involving a woman's social network in postpartum care may add value to care for this population. In addition, by addressing a woman's self-efficacy and social network in the different phases of the process of postpartum care, care utilisation can potentially be improved. The conceptual framework generated in this study may also be used as a basis to optimise care utilisation for vulnerable pregnant and postpartum women outside the Netherlands.

Acknowledgements We acknowledge the pregnant and postpartum women for participating in this study. We thank the participating healthcare providers for approaching the women. Many thanks to Lisanne Vermeulen, Hajrah Arshad, Karishma Haripersad and Sin Mok for the transcriptions of the interviews.
Contributors LTL, JL, HEE-S, JVB, AF and ML designed the study. LTL performed the data collection. LTL and MvdH analysed the data under supervision of ML. All authors interpreted the data. LTL, MvdH, JL and ML wrote the first version of the manuscript. All authors revised all versions of the manuscript and approved the final version.

Funding This study was funded by the Netherlands Organisation for Health Research and Development (ZonMw), grant number 209070002.

Competing interests None declared.

Patient consent for publication Not required.

Ethics approval The Medical Ethics Committee of the Erasmus University Medical Centre declared that the Medical Research Involving Human Subjects Act did not apply to this study and approved the study protocol (MEC-2017-1081). Participation was on a voluntary basis and all women participating in the study provided written informed consent.

Provenance and peer review Not commissioned; externally peer reviewed.

Data availability statement Data are available upon reasonable request.

Supplemental material This content has been supplied by the author(s). It has not been vetted by BMJ Publishing Group Limited (BMJ) and may not have been peer-reviewed. Any opinions or recommendations discussed are solely those of the author(s) and are not endorsed by BMJ. BMJ disclaims all liability and responsibility arising from any reliance placed on the content. Where the content includes any translated material, BMJ does not warrant the accuracy and reliability of the translations (including but not limited to local regulations, clinical guidelines, terminology, drug names and drug dosages), and is not responsible for any error and/or omissions arising from translation and adaptation or otherwise.

Open access This is an open access article distributed in accordance with the Creative Commons Attribution Non Commercial (CC BY-NC 4.0) license, which permits others to distribute, remix, adapt, build upon this work non-commercially, and license their derivative works on different terms, provided the original work is properly cited, appropriate credit is given, any changes made indicated, and the use is non-commercial. See: http://creativecommons.org/licenses/by-nc/4.0/.

\section{ORCID iDs}

Lyzette T Laureij http://orcid.org/0000-0001-6342-7528

Jacqueline Lagendijk http://orcid.org/0000-0002-7178-8910

\section{REFERENCES}

1 Bryant AS, Haas JS, McElrath TF, et al. Predictors of compliance with the postpartum visit among women living in healthy start project areas. Matern Child Health J 2006;10:511-6.

2 Waelput AJM, Sijpkens MK, Lagendijk J, et al. Geographical differences in perinatal health and child welfare in the Netherlands: rationale for the healthy pregnancy 4 all-2 program. BMC Pregnancy Childbirth 2017;17:254.

3 Mackenbach JP. The persistence of health inequalities in modern welfare states: the explanation of a paradox. Soc Sci Med 2012;75:761-9.

4 Scheele J, Harmsen van der Vliet-Torij HW, Wingelaar-Loomans EM, et al. Defining vulnerability in European pregnant women, a Delphi study. Midwifery 2020;86:102708.

5 Briscoe L, Lavender T, McGowan L. A concept analysis of women's vulnerability during pregnancy, birth and the postnatal period. $J A d v$ Nurs 2016;72:2330-45.

6 de Groot N, Bonsel GJ, Birnie E, et al. Towards a universal concept of vulnerability: broadening the evidence from the elderly to perinatal health using a Delphi approach. PLoS One 2019;14:e0212633.

7 Pillas D, Marmot M, Naicker K, et al. Social inequalities in early childhood health and development: a European-wide systematic review. Pediatr Res 2014;76:418-24.

8 de Graaf JP, Ravelli ACJ, de Haan MAM, et al. Living in deprived urban districts increases perinatal health inequalities. J Matern Fetal Neonatal Med 2013;26:473-81.

9 de Graaf JP, Steegers EAP, Bonsel GJ. Inequalities in perinatal and maternal health. Curr Opin Obstet Gynecol 2013;25:98-108.

10 World Health Organization. WHO recommendations on postnatal care of the mother and newborn. World Health Organization, 2014.

11 Verbiest S, Bonzon E, Handler A. Postpartum health and wellness: a call for quality Woman-Centered care. Matern Child Health $J$ 2016;20:1-7.

12 Wiegers TA. Adjusting to motherhood: maternity care assistance during the postpartum period: how to help new mothers cope. Journal of Neonatal Nursing 2006;12:163-71. 
13 Baas Cl, Wiegers TA, de Cock TP, et al. Experience with and amount of postpartum maternity care: Comparing women who rated the care they received from the maternity care assistant as 'good' or 'less than good care'. Midwifery 2017:55:128-36.

14 Lagendijk J, Steegers EAP, Been JV. Inequity in postpartum healthcare provision at home and its association with subsequent healthcare expenditure. Eur J Public Health 2019;29:849-55.

15 Shaw E, Levitt C, Wong S, et al. Systematic review of the literature on postpartum care: effectiveness of postpartum support to improve maternal parenting, mental health, quality of life, and physical health. Birth 2006;33:210-20.

16 Strauss A, Corbin J. Basics of qualitative research techniques. Thousand Oaks, CA: Sage publications, 1998.

17 Glaser BG, Strauss AL. Discovery of grounded theory: strategies for qualitative research. Routledge, 2017.

18 Casp. Critical appraisal skills programme, CASP (qualitative) checklist, 2013.

19 Green J, Thorogood N. Qualitative methods for health research. Sage, 2018.

20 O'Brien BC, Harris IB, Beckman TJ, et al. Standards for reporting qualitative research: a synthesis of recommendations. Acad Med 2014;89:1245-51.

21 UyBico SJ, Pavel S, Gross CP. Recruiting vulnerable populations into research: a systematic review of recruitment interventions. J Gen Intern Med 2007;22:852-63.

22 Murthy VH, Krumholz HM, Gross CP. Participation in cancer clinical trials: race-, sex-, and age-based disparities. JAMA 2004;291:2720-6.

23 Bonevski B, Randell M, Paul C, et al. Reaching the hard-to-reach: a systematic review of strategies for improving health and medical research with socially disadvantaged groups. BMC Med Res Methodol 2014;14:42.

24 Kuper A, Reeves S, Levinson W. An introduction to reading and appraising qualitative research. BMJ 2008;337:a288.

25 Walker SB, Rossi DM, Sander TM. Women's successful transition to motherhood during the early postnatal period: a qualitative systematic review of postnatal and midwifery home care literature. Midwifery 2019;79:102552.

26 Cutrona CE, Troutman BR. Social support, infant temperament, and parenting self-efficacy: a mediational model of postpartum depression. Child Dev 1986;57:1507-18

27 Rayment-Jones $\mathrm{H}$, Harris J, Harden A, et al. How do women with social risk factors experience United Kingdom maternity care? A realist synthesis. Birth 2019;46:461-74.

28 Guerra-Reyes L, Christie VM, Prabhakar A, et al. Postpartum health information seeking using mobile phones: experiences of low-income mothers. Matern Child Health J 2016;20:13-21.
29 Slomian J, Emonts P, Vigneron L, et al. Identifying maternal needs following childbirth: a qualitative study among mothers, fathers and professionals. BMC Pregnancy Childbirth 2017;17:213.

30 Laureij LT, Breunis LJ, Steegers-Theunissen RPM, et al. Identifying the needs for a web-based postpartum platform among parents of newborns and health care professionals: qualitative focus group study. JMIR Form Res 2020;4:e16202.

31 Goto E, Ishikawa H, Okuhara $\mathrm{T}$, et al. Relationship of health literacy with utilization of health-care services in a general Japanese population. Prev Med Rep 2019;14:100811.

32 Berkman ND, Sheridan SL, Donahue KE, et al. Low health literacy and health outcomes: an updated systematic review. Ann Intern Med 2011;155:97-107.

33 Noordman J, van Vliet L, Kaunang M, et al. Towards appropriate information provision for and decision-making with patients with limited health literacy in hospital-based palliative care in Western countries: a scoping review into available communication strategies and tools for healthcare providers. BMC Palliat Care 2019;18:37.

34 Finlayson $\mathrm{K}$, Crossland $\mathrm{N}$, Bonet $\mathrm{M}$, et al. What matters to women in the postnatal period: a meta-synthesis of qualitative studies. PLoS One 2020;15:e0231415

35 Xiao X, Ngai F-W, Zhu S-N, et al. The experiences of early postpartum Shenzhen mothers and their need for home visit services: a qualitative exploratory study. BMC Pregnancy Childbirth 2019;20:5.

36 Blanchard J, Lurie N. R-E-S-P-E-C-T: patient reports of disrespect in the health care setting and its impact on care. J Fam Pract 2004:53:721-30.

37 Lindquist A, Kurinczuk JJ, Redshaw M, et al. Experiences, utilisation and outcomes of maternity care in England among women from different socio-economic groups: findings from the 2010 national maternity survey. BJOG 2015;122:1610-7.

38 McLeish J, Redshaw M. Maternity experiences of mothers with multiple disadvantages in England: a qualitative study. Women Birth 2019;32:178-84.

39 Plomp HN, Ballast N. Trust and vulnerability in doctor-patient relations in occupational health. Occup Med 2010;60:261-9.

40 Goodarzi B, Walker A, Holten L, et al. Towards a better understanding of risk selection in maternal and newborn care: a systematic scoping review. PLoS One 2020;15:e0234252.

41 Balaam M-C, Thomson G. Building capacity and wellbeing in vulnerable/marginalised mothers: a qualitative study. Women Birth 2018;31:e341-7.

42 UIf S. International standard classification of education: ISCED 2011: UNESCO Institute for statistics Montreal, 2012 